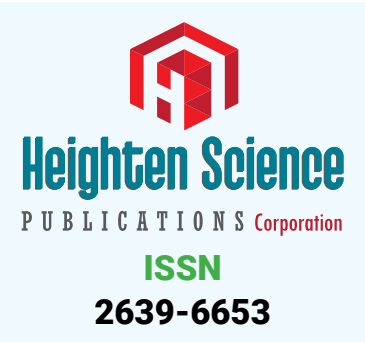

*Address for Correspondence: Igor Klepikov, MD Professor, 2116, NE, 27 St., Renton, WA, 98056, USA, Email: igor.klepikov@yahoo.com

Submitted: 08 March 2018

Approved: 15 March 2018

Published: 16 March 2018

Copyright: @ 2018 Klepikov I. This is an open access article distributed under the Creative Commons Attribution License, which permits unrestricted use, distribution, and reproduction in any medium, provided the original work is properly cited

A) Check for updates
Letter to Editor

\section{Dependence of the results of treatment of acute pneumonia on the doctrine of the disease}

\author{
Igor Klepikov* \\ Professor, Renton, WA, USA
}

\section{Introduction}

Treatment of various inflammatory processes, including acute pneumonia(AP), over the past decades is identical and does not reflect the specifics of a particular disease. The basis of such treatment is «antibiotics alone». The need for additional therapeutic efforts is realized by the use of General therapeutic techniques, regardless of the diagnosis. This does not take into account the important fact that the localization of inflammation not only determines its clinical picture,but,above all,the mechanisms of influence on other organs and systems of the body.

Attempts to cure fundamentally different diseases with the help of one aid package cannot give the expected result even theoretically. The futility of this approach is illustrated by the current dynamics of the results.Reducing the effectiveness of antibiotics and the emergence of new resistant strains of microorganisms forces the constant development of new antimicrobials. However, the success of pharmacists can not stop the continued increase in the number of purulent complications of AP even in developed countries with maximum capacity to provide patients. There is a solution to the existing problem and it lies in another plane.

First results guaranteed prophylaxis of the complicated course AP was received in the years 1976-1984 in Novokuznetsk State Institute for postgraduate doctors(USSR,Russia). This work not only included original studies, but was also tested in clinical practice. The main feature of this work is a complete revision of the system of views on the nature of AP and the creation of a new doctrine of the disease. For this purpose already known scientific facts were demanded.

1. AP is not a contagious specific disease. Non-specific pathogens of AP can occur among symbionts of healthy people.

2. The body's reaction to the development of the inflammatory process and its compensatory capabilities have a strictly individual range.

3. Inflammatory transformation of body tissues is caused by vascular reaction, which has known successive stages

4. Small and big circles of blood circulation have a direct anatomical and functional interdependence.

5. The vessels of the lesser circulation are highly sensitive reflexogenic zone.

6. Acute pneumonia is the only process among other non-specific forms of inflammation that develops in the vascular system of the small circle of blood circulation. 
7. The effect of the same medical procedure on a small and large circle of blood circulation can have different consequences, since their functional relationship has a feedback dependence. At the same time, any intravenous therapy should take into account the fact that the zone of inflammation in the lung is the first barrier to administered drugs.

The following additional studies were carried out to clarify some details of the occurrence and dynamics of AP.

1. Experimental model of AP (4 series of experiments, 44 animals) with targeted obtaining of pleural complications (the patent for the invention No 1631574, A1, 1 November 1990, USSR.).

2. X-ray contrast study of 56 pulmonary anatomical preparations with different forms of AP taken from deceased patients.

3. Record comparative rheopulmonography before and after performing medical procedures(36 patients).

4. Analysis of supervision and treatment of 994 children with different initial forms of AP and difference in treatment principles [1].

The already known scientific information and the obtained additional results made it possible to look at the nature and pathogenesis of acute pneumonia from other positions. Preliminary sensitization of the body plays an important role in the occurrence of the disease. Repeated absorption of allergen in the lung tissue in combination with symbionts triggers the inflammatory process. Local inflammatory changes of pulmonary vessels cause reflex reaction and generalized spasm of blood vessels of small circle. The speed of development of this process has an individual characteristic and is subject to counteraction of adaptive opportunities of an organism. The slower the inflammatory process develops, the more opportunities the body has to minimize its manifestations.Diagnosis of the disease in such cases can cause difficulties,and treatment may be late. However, the use of antibiotics only and the suppression of raging microflora allows the body to get out of this situation without problems.

A different picture is observed in the case of aggressive development of the disease. The inability to quickly adapt to the violation of perfusion in the lungs immediately causes the restructuring of blood circulation in a large circle. The nature and severity of such violations in the most difficult situations fully corresponds to the clinical picture of shock. This form of shock was described by us previously as pulmonary shock [2]. Thus,it is necessary to make a very important clarification that acute pneumonia is more circulatory than respiratory catastrophe.

The combination of clinical observations and various studies allowed to speak about the negative impact of intravenous infusions on the dynamics of acute pneumonia. The result of this conclusion was the exclusion of intravenous infusion of solutions from the complex of first aid for pneumonia.

Other treatments were used as first aid to newly admitted patients.Such first-aid techniques (cervical vagosympathetic blockade, cupping therapy, patient body cooling) were considered by us as anti-shock activity. The positive effect of these procedures was confirmed by objective evaluation criteria.Further treatment included antibiotic therapy and active restoration of bronchial patency and airiness of the lung.

The revised principles of treatment were applied in 101 patients in the initial period of aggressive forms of AP.In all these observations, the process was quickly and completely resolved without any complications. 
For comparison, it is very significant to present the results of treatment of 102 children who already had effusion in the pleural cavity at the time of hospitalization. The presence of pleural effusion have testified to a deeper degree of inflammatory changes. In this group of patients, the inflammatory process in the lung was also eliminated quickly enough. However, the process of resolving inflammatory infiltration in 52 patients was accompanied by the appearance of destructive cavities. The appearance of cavities was characteristic of patients who, at the time of hospitalization, had a more viscous fibrinous effusion. Therefore, the presence and quality of pleural effusion we regarded as an indicator of the severity of the initial inflammatory changes. This feature allows to predict the final result of treatment.

A more detailed presentation and justification of the new doctrine AP, the results of these studies and clinical trials can be found in the published book: Igor Klepikov"Acute pneumonia: a new look at the old problem",Lambert Academic Publishing,2017,IS $B N(978-3-330-35250-6)$.

The obtained results indicate the possibility of influencing the mechanisms of AP development both in the direction of stimulation and inhibition. Timely inhibition of the dynamics of the inflammatory process involves guaranteed prevention of purulent and destructive complications of the disease.

\section{References}

1. Klepikov I. "Acute pneumonia and its purulent and destructive complications in children in the midst of a major industrial centre of Western Siberia». Dissertation for the degree of doctor of medical sciences.Leningrad. 1989.

2. Klepikov I, Yudin J. "Shock in cases of acute bronchogenic pneumonia."- A collection of articles "Clinical and anatomical aspects of disseminated intravascular blood coagulation and shock". Leningrad, 1986, Leningrad Institute for Advanced Training of Doctors. 1986: 53-55. 